\title{
A Comparison of Thioredoxin 1 Gene Expression in Schizophrenia Patients before and after Treatment with Risperidone
}

\author{
ESFANDIAR AZIZI ${ }^{1}$, MORTEZA HOSSEINZADEH ${ }^{* 1}$, PARVIZ VAHDATIAN ${ }^{1}$, AMIR ADIBI $^{2}$, AKBAR AZIZIFAR ${ }^{3}$, HOJAT \\ SAYYADI ${ }^{4}$, OMRAN DAVARINEJAD ${ }^{5}$, HOSSEIN SEIDKHANI ${ }^{6}$, KARIMEH HAGHANI ${ }^{7}$ \\ ${ }^{1}$ Department of Immunology, School of Medicine, Ilam University of Medical Sciences, Ilam, Iran. \\ ${ }^{2}$ Department of Child and Adolescent Psychiatry, School of Medicine, Ilam University of Medical Sciences, Ilam, Iran. \\ ${ }^{3}$ Department of Psycholinguistics, School of Medicine, Ilam University of Medical Sciences, Ilam, Iran. \\ ${ }^{4}$ Department of Biostatistics, School of Health, Ilam University of Medical Sciences, Ilam, Iran. \\ ${ }^{5}$ Department of Psychiatry, School of Medicine, Kermanshah University of Medical Sciences, Kermanshah, Iran. \\ ${ }^{6}$ Department of Biostatistics, School of Health, Ilam University of Medical Sciences, Ilam, Iran. \\ ${ }^{7}$ Department of Clinical Biochemistry, School of Medicine, Ilam University of Medical Sciences, Ilam, Iran. \\ Correspondence to: * MORTEZA HOSSEINZADEH, Department of Immunology, School of Medicine, Ilam University of Medical Sciences, \\ Ilam, Iran.E-mail address: Hosseinzadeh-m@medilam.ac.ir; mortezahoseinzadeh@hotmail.com.
}

\begin{abstract}
Background: Schizophrenia is a mental disorder characterized by distortions in thinking, perception, emotions, language, self-sense, and behavior. Recent research suggests that Reactive Oxygen Species (ROS) are involved in the pathophysiology of schizophrenia. Studies have also shown the increased plasma and serum levels of the Trx1 molecule in schizophrenia patients. In the present study, the researchers compared the expression levels of Trx1 mRNA in peripheral blood leukocytes of Iranian schizophrenia patients compared to healthy controls.

Methods: First-episode patients $(n=35)$ who met DSM-IV criteria for schizophrenia were recruited from patients referred to psychiatrists in the city of Ilam and Farabi Hospital in Kermanshah. Healthy people were also selected by recruiting people who, according to a psychiatrist, did not have any mental illness. Diagnoses were made for each patient by two independent experienced psychiatrists and confirmed by the Structured Clinical Interview for DSM-IV (SCID). Patients were treated with risperidone for three months and then compared with thirty- five healthy volunteers. Patients were sampled before and after treatment and then by RNA Extraction and DNA synthesis, Trx1 gene expression was performed by real-time PCR method.

Results: Comparison of Trx1 gene expression in PBMCs of schizophrenic patients before and after treatment with the control group showed that the expression of Trx1 gene of the "before" treatment group was significantly increased compared with that of the control group $(P=0.0007)$. Also, Trx1 gene expression in PBMCs of "before" and "after "groups showed that Trx1 gene expression of "after" group was significantly decreased compared to the "before" group $(P=0.014)$. These results showed that the mean of positive, negative, and general psychopathology was reduced significantly in schizophrenic patients before and after treatment in all three cases $(\mathrm{P}<0.001)$.

Conclusion: the expression of TRX in PBMCs of schizophrenic patients decreased after risperidone treatment. This reduction of expression was statistically significant and indicates the possible effect of risperidone on the expression of the TRX gene in PBMCs of these patients and decreased gene expression is associated with reduced symptoms. Confirmation of the achievement of this study requires further research.

Key words: Schizophrenia, Thioredoxin, Risperidone
\end{abstract}

\section{INTRODUCTION}

Schizophrenia is a mental disorder characterized by distortions in thinking, perception, emotions, language, self sense, and behaviour [1]. The etiopathology of schizophrenia remains unknown. A combination of multiple factors such as genetic background, inflammation, viral infections, environmental factors, etc., might be implicated in schizophrenia pathogenesis [2, 3]. Recent research suggests that reactive oxygen species (ROS) are involved in the pathophysiology of schizophrenia [4]. Antioxidant systems control the level of ROS, oxidative stress can take place if ROS levels exceed the antioxidant capabilities, and this can have harmful functional effects for cells. For example, ROS destroy lipid membranes by peroxidation of fatty acids and trigger various pathological processes [5]. The thioredoxin system is one of the major antioxidant systems applied by cells to defend against oxidative stress [6]. Thioredoxin (Trx) molecule, Thioredoxin Reducates (TrxR), and NADPH are components of the Trx / TrxR system; this system is one of the key regulators of the redox system, which plays a key role in many intracellular activities [7], also the Trx molecule plays a critical role in immune responses, viral infection, and cell death through its interaction with its binding protein [8]. In mammalian cells, there are two isoforms of $\operatorname{Trx}$ (Trx1 and $\operatorname{Trx} 2)$. Thioredoxin 1 (Trx1) is mainly located in the cytoplasm, it is ubiquitously expressed in a variety of cells and control cellular ROS by reducing the disulfides into thiol groups [9]. The thioredoxin system has been reported to be disrupted in various diseases [9]. Studies have also shown increased plasma and serum levels of the Trx molecule in schizophrenia patients [10]. In the present study, we compared the expression levels of Trx mRNA in peripheral blood leukocytes of Iranian schizophrenia patients (in two groups, before and after treatment with risperidone) compared to healthy controls.

\section{METHODS AND MATERIALS}

Subjects: First-episode patients (assigned as the before treatment $(\mathrm{BT})$ group $)(\mathrm{N}=35$; males $=23$ and 
females=12) who met DSM-IV criteria for schizophrenia were recruited from patients referred to psychiatrists in Taleghani Hospital in Ilam and Farabi Hospital in Kermanshah. Healthy people were also selected by recruiting people who, according to a psychiatrist, did not have any mental illness. Diagnoses were made for each patient by two independent experienced psychiatrists and confirmed by the Structured Clinical Interview for DSM-IV (SCID). Patients were treated with risperidone for three months based on each patient's condition (assigned as the after-treatment (AT) group) and compared with thirty- five healthy volunteers (assigned as the Normal controls (NC) group). The age and sex of the control group and patients' group were matched. In the control group, those who received corticosteroids, immunosuppressive, chronic Immune illness, and psychotropic drugs, as well as those with a family history of mental illness were not included in the study. The exclusion criteria for patient groups were history of head trauma, mental retardation, use of any corticosteroids and immunosuppressive, history of substance abuse, chronic immune illness is known to be accompanied by changes in immune cell functions. The positive and negative symptoms of both patients (BT and AT) groups were assessed using a Positive and Negative Syndrome Scale (PANSS). Demographic data for patients and normal controls are summarized in Table 1. The study was approved by the Ethics Committee of Ilam University of Medical Sciences (Ilam, Iran), and all participants and/or legal representatives provided written informed consent.

Sampling and Separation of peripheral blood mononuclear cells (PBMCs): Patients (BT) sampling was done with the coordination of the treating physician and patient legal representative satisfaction. Sampling was repeated three months after the start of treatment in the patient (AT) group under previous conditions; also normal group sampling was performed with the consent of the Participants. $10 \mathrm{mls}$ of venous blood samples from each individual in each group were collected in tubes containing ethylene di-amide tetra acetic acid (EDTA) anticoagulant (Merck, Darmstadt, Germany) under sterile conditions. The samples were quickly transferred to the research laboratory. Peripheral blood mononuclear cells (PBMCs) were isolated by performing a Ficoll-Paque ${ }^{\mathrm{TM}}$ PLUS gradient (GE Healthcare, UK) according to the standard protocol. Then, the PBMCs layer was removed and transferred to $1.5 \mathrm{ml}$ RNase free microtubules (Ratiolab
$\mathrm{GmbH}$, Dreieich, Germany) and total RNA extraction was performed immediately.

Total RNA extraction and cDNA synthesis: The total RNA was extracted from the PBMC cells of the test and control groups by a Trizol reagent (Gibco BRL) according to the manufacturer's instructions. The total RNA concentration and purity were determined with the NanoDrop 2000 (Thermo Scientific, MA) and stored at $-80^{\circ} \mathrm{C}$ until the cDNA synthesis was performed. Total RNA was reversely transcribed into cDNA using the Thermo Scientific Revert Aid First Strand cDNA Synthesis Kit (\#K1622) as described in the manufacturer's protocol.

Real-time polymerase chain reaction (PCR) assay: To verify the presence of cDNA and quality control of the samples as well as to obtain the appropriate temperature profile for the target genes, several randomly generated cDNA samples were selected and the PCR reaction was performed in a special thermocycler (Eppendorf Mastercycler gradient, Biotech Co., Germany) using a kit (RealQ Plus 2x Master Mix Green) and according to the kit manufacturer's instructions (Amplicon, Denmark). The mRNA expression of the TRX1 gene was then measured by relative quantitative real-time PCR using SYBR ${ }^{\circ}$ Green Master Mix-Plus (Amplicon, Odense, Denmark) as guided by the manufacturers, pairs of gene-specific primers and a real-time PCR thermocycler (Corbett RG6000, Australia). Real-time PCR reactions were performed in duplicates in a final volume of $20 \mu \mathrm{L}$ containing $10 \mu \mathrm{l}$ DNA polymerase mater mix, $1 \mu \mathrm{L}$ primer mix (2 pmol/mL) and $5 \mathrm{ng}$ of cDNA. The net volume was reached to $20 \mu \mathrm{l}$ by adding Double distilled DNase free water. Oligonucleotides were synthesized by Macrogen (South Korea) which are shown in (Table 1). Real-time PCR was performed in duplicates starting with a denaturation phase at $95{ }^{\circ} \mathrm{C}$ for $15 \mathrm{~min}$, followed by 40 three-step cycles of denaturation at $95{ }^{\circ} \mathrm{C}$ for $10 \mathrm{~s}$, annealing at $55-58^{\circ} \mathrm{C}$ for $10 \mathrm{~s}$ and extension at 72 for $30 \mathrm{~s}$. The reaction was terminated in a dissociation stage for recording the product melt. To assay the quality of the reaction product, DNA samples were loaded onto agarose gel $2 \%$ and electrophoresed at $75 \mathrm{~V}$ for $40 \mathrm{~min}$ and they were detected using a gel doc machine and confirmed to be a single band. The relative expression levels were normalized $(2 \Delta \mathrm{ct})$ to $\beta$-Actin as the endogenous control. The primers used for the Trx and $\beta$ Actin genes are listed in Table $2[11,12]$.

Table 1: Oligonucleotide sequences of the primers designed for Real-time PCR.

\begin{tabular}{|c|c|c|c|}
\hline Oligonucleotide name & Product name & Sequence $\left(5^{\prime}-3^{\prime}\right)$ & Connection temperature $\left(\mathrm{C}^{\circ}\right)$ \\
\hline \multirow{2}{*}{ Trx1 } & Forward primer & 5'-TGGTGAAGCAGATCGAGAGCAAGA-3' & \multirow{2}{*}{58} \\
\hline & Reverse primer & 5'ACCACGTGGCTGAGAAGTCAACTA-3' & \\
\hline \multirow{2}{*}{$\beta$-Actin } & Forward primer & 5'-CCTGGGCATGGAGTCCTGT-3' & \multirow[b]{2}{*}{56} \\
\hline & Reverse primer & 5'-ATCTCCTTCTGCATCCTGTCG-3' & \\
\hline
\end{tabular}

Statistical Analyses: The results are described using the mean, standard deviation (SD), and standard error measurment (SEM). The normal distribution of data was tested using the Shapiro-Wilk and the KolmogorovSmirnov tests; assuming the normality of data was accepted. One-way analysis was used for significant differences between means of Trx1 gene expression in peripheral blood cells in the three study groups, and thereafter, pairwise T-tests were performed for post hoc comparisons. The psychopathology on the PANSS was compared between the patient groups by one-way ANOVA and correlated with expression of Trx1 level within the patient groups by calculating Pearson correlation coefficients. Differences at a level of $p<0.05$ were considered to be significant. 
RESULTS AND DISCUSSION

Demographic data: Table 2 shows the demographic characteristics of the patient and control group participants.

The results showed that in the patient and control groups, the mean age of onset was $29.40 \pm 5.81$ and $29.48 \pm 5.23$ years respectively. The positive and negative symptoms of the schizophrenia groups were assessed using a Positive and Negative Syndrome Scale (PANSS).

Table 2. Demographic characteristics and PANSS symptoms scale of the patients before and after treatment with risperidone and Normal controls

\begin{tabular}{|l|l|l|l|l|}
\hline \multirow{2}{*}{ Parameter } & Patient & \multirow{2}{*}{ Normal controls } & \multirow{2}{*}{$p$-Value } \\
\cline { 2 - 4 } & Before treatment & After treatment & & \\
\hline Sex & & & & \\
\hline Male & $25(71.42 \%)$ & $25(71.42 \%)$ & $23(65.71 \%)$ \\
\hline Female & $10(28.58 \%)$ & $10(28.58 \%)$ & $12(34.29 \%)$ & \\
\hline Age (years, mean \pm SD) & $29.40 \pm 5.81$ & $29.40 \pm 5.81$ & $29.48 \pm 5.23$ & \\
\hline Positive symptoms (mean \pm SD) & $22.71 \pm 4.61$ & $15.02 \pm 5.32$ & - & 0.000 \\
\hline Negative symptoms (mean \pm SD) & $19.42 \pm 4.12$ & $13.91 \pm 5.09$ & - & 0.002 \\
\hline General psychopathology (mean \pm SD) & $42.48 \pm 6.58$ & $30.08 \pm 5.47$ & - & 0.000 \\
\hline PANSS total score & $84.61 \pm 15.31$ & $59.01 \pm 15.88$ & - & 0.000 \\
\hline
\end{tabular}

* The descriptive statistical results were expressed as a mean \pm standard deviation. A significant p-value calculated using Independent Samples T-test and the statistical significance level was set at $p<0.05$. PANSS: Positive and Negative Syndrome Scale.

As shown in Table 2, there is a significant difference between positive and negative symptoms, general psychopathology and PANSS total score in the before and after patients' treatment are $(P=0.000, P=0.002, P=0.000$ and $\mathrm{P}=0.000$ ) respectively.

Evaluation results of Trx1 gene expression in peripheral blood cells: Comparison of Trx1 gene expression in PBMCs of schizophrenic patients before and after treatment with the control group (Table 3) showed that the expression of Trx1 gene in PBMCs of the BT group was significantly increased compared with that of the control group (P-value $=0.0007)$. Despite the increase in Trx1 gene expression in PBMCs of AT group compared to that of the control group, this difference was not statistically significant $(P$-value $=0.0570)$.

Table 3: Comparison of Trx1 gene expression in PBMCs of schizophrenia patients before and after treatment compared to the control group

\begin{tabular}{|l|l|l|l|l|l|}
\hline Variable & Obs & Mean & STd. Err & STd. Dev & [95\%. Conf. interval] \\
\hline$\Delta$ CT-BT & 35 & 11.74857 & 0.360323 & 2.131702 & 11.01631 \\
$\Delta$ CT-Control & 35 & 14.15143 & 0.565946 & 3.348186 & 13.00129 \\
Combined & 70 & 12.95 & 0.363071 & 3.03767 & 12.22569 \\
Diff & & -2.402857 & 0.670916 & 15.36157 \\
\hline$\Delta$ CT-AT & 35 & 12.89843 & 0.312584 & 13.67431 & -3.746004 \\
$\Delta$ CT-Control & 35 & 14.15143 & 0.565946 & 1.849275 & -13.00129 \\
Combined & 70 & 13.52243 & 0.329728 & 3.348186 & 13.5286711 \\
Diff & -1.258 & 0.6465329 & 2.758703 & 15.30157 \\
\hline
\end{tabular}

$\begin{array}{ll}\text { Two-Sample T-Test with unequal Variances } & \text { BT: Before treatment group } \\ \mathrm{P}(\Delta \mathrm{CT}-\mathrm{BT} / \text { Control })=0.0007 & \text { AT: After treatment group }\end{array}$

$\mathrm{P}(\Delta \mathrm{CT}-\mathrm{AT} /$ Control $)=0.0570$

Table 4: Comparison of Trx1 gene expression in PBMCs of schizophrenic patients before and after treatment

Table 4: Comparison of Trx1 gene expression in PBMCs of schizophrenic patients before and after treatment
\begin{tabular}{|l|l|l|l|l|l|}
\hline Variable & Obs & Mean & STd. Err & STd. Dev & [95\%. Conf. interval] \\
\hline$\Delta \Delta$ CT-BT & 35 & -2.402857 & 0.360323 & 2.131702 & -3.135122 \\
$\Delta \Delta$ CT-AT & 35 & -1.258 & 0.312584 & 1.849275 & -1.893248 \\
Diff & 35 & -1.144857 & 0.123389 & 0.729981 & -1.395615 \\
\hline FC-BT & 35 & 16.18207 & 5.803239 & 34.33243 & -0.622751 \\
FC-AT & 35 & 5.426275 & 1.477035 & 8.738259 & -0.894099 \\
Diff & 35 & 10.7558 & 4.670374 & 27.6303 & 2.424578 \\
\hline
\end{tabular}

Paired T-Test BT: Before treatment group

$\mathrm{P}(\mathrm{DDCT}-\mathrm{BT} / \mathrm{AT})=0.000 \quad \mathrm{AT}:$ After treatment group

$\mathrm{P}(\mathrm{FC}-\mathrm{BT} / \mathrm{AT})=0.014 \quad$ FC: Fold change

Table 5: Comparison of positive symptom, negative symptoms and General Psychopathology in

schizophrenia patients before and after treatment

\begin{tabular}{|l|l|l|l|l|l|l|}
\hline Variable & Obs & Mean & STd. Err & STd. Dev & [95\%. Conf. interval] \\
\hline PS - BT & 35 & 22.71429 & 0.952282 & 5.633782 & 20.77901 \\
PS - AT & 35 & 15.02857 & 0.768591 & 4.547046 & 13.46661 \\
Diff & 35 & 7.685714 & 0.883814 & 5.228718 & 5.889587 \\
\hline NS -BT & 35 & 19.42857 & 0.8795393 & 5.203425 & 16.59054 \\
NS -AT & 35 & 13.91429 & 0.7360711 & 4.354656 & 9.481842 \\
Diff & 35 & 5.514286 & 0.95387 & 5.643171 & 12.41841 \\
\hline GP-BT & 35 & 42.48571 & 1.406092 & 8.318553 & 3.575789 \\
GP-AT & 35 & 30.08571 & 1.127481 & 6.670265 & 39.62819 \\
Diff & 35 & 12.4 & 1.594212 & 9.431487 & 27.7944 \\
\hline
\end{tabular}

Paired t-test

$\mathrm{P}<0.001$
PS: Positive Symptoms NS: Negative Symptoms
1.594212

GP: General Psychopathology

AT: After treatment group
BT: Before treatment group
: Before treatment group 
Table 6: Correlation of the studied variables in patients with schizophrenia before and after treatment

\begin{tabular}{|c|c|c|c|c|c|}
\hline & Age & Diff-GP & Diff-FC & Diff-PS & Diff-NS \\
\hline Age & 1.000 & & & & \\
\hline Diff-GP & $\begin{array}{l}-0.0501 \\
0.7751\end{array}$ & 1.000 & & & \\
\hline Diff-FC & $\begin{array}{l}0.3066 \\
0.0733\end{array}$ & $\begin{array}{l}-0.2294 \\
0.1849\end{array}$ & 1.000 & & \\
\hline Diff-PS & $\begin{array}{l}-0.1398 \\
0.4230\end{array}$ & $\begin{array}{l}0.0420 \\
0.8107\end{array}$ & $\begin{array}{l}-0.0099 \\
0.9550\end{array}$ & 1.000 & \\
\hline Diff-NS & $\begin{array}{l}0.0323 \\
0.8536\end{array}$ & $\begin{array}{l}0.1386 \\
0.4272\end{array}$ & $\begin{array}{l}-0.1728 \\
0.3209\end{array}$ & $\begin{array}{l}0.4681 \\
0.0046^{*}\end{array}$ & 1.000 \\
\hline
\end{tabular}

PS: Positive Symptoms $\quad$ GP: General Psychopathology

NS: Negative Symptoms FC: Fold change

Analysis of Trx1 gene expression in PBMCs of BT and AT groups (Table 4) showed that Trx1 gene expression in PBMCs of AT group was significantly decreased compared to the BT group $(P$-value $=0.014)$.

Correlation between Trx1 gene expression in peripheral blood cells and positive and negative syndrome scale (PANSS): Comparisons among positive symptoms, negative symptoms, and general psychopathology were also performed in patients with schizophrenia before and after treatment (results are shown in Table 5). These results showed that the mean of positive symptoms in patients before treatment (22.71) compared to after treatment (15.02) was reduced. Also, the mean of negative symptoms and general psychopathology in patients before treatment compared to after treatment decreased from 19.42 to 13.91 and from 42.48 to 30.08 , respectively and in all three cases, this decrease and difference were statistically significant $(P<0.001)$. Also, the correlation of the studied variables in schizophrenia patients before and after treatment was examined and the diagram (No 1) Matrix Scatter Plot shows this correlation.
This diagram shows the correlation between negative symptoms and positive symptoms so that the reduction of negative symptoms after treatment of patients is associated with a decrease in positive symptoms after treatment $(p=0.0046)$, but this decrease is not correlated with the expression of the Trx1 gene before and after treatment (Table 6). Of course, in another analysis, the correlation of the studied variables in patients with schizophrenia and after treatment was also performed, but in this analysis no significant relationship was observed, but two important points are to be considered; first, the correlation between gene expression (FC) after treatment with positive symptoms of the disease was -0.31 , which is not significant $(P=0.07)$, but shows how much Trx1 gene expression is after less treatment, the positive symptoms of the disease have also decreased. Another correlation is the correlation between the expression of the Trx1 (FC) gene after treatment with negative symptoms of the disease, which is equal to $-0.28(P=0.09)$, which again indicates a decrease in negative symptoms associated with a decrease in the Trx1 gene expression (Results are not shown).

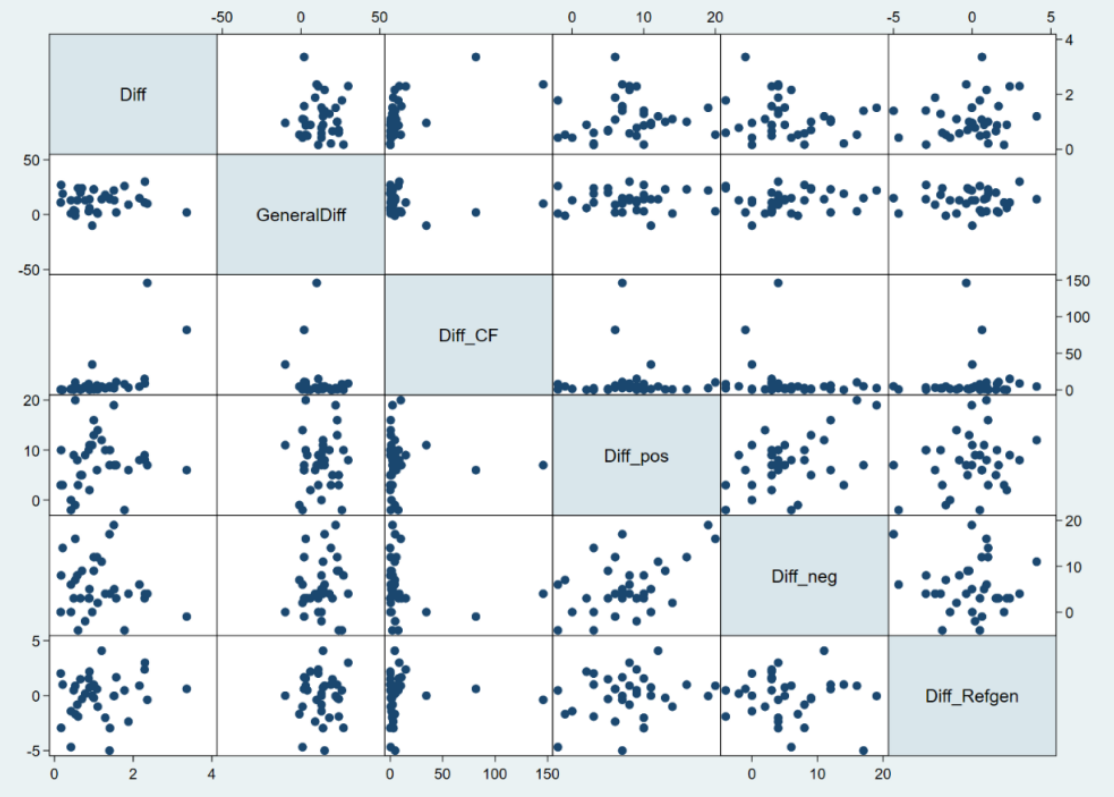

Diagram 1: Matrix Scatter Plot shows the correlation of the studied variables in schizophrenia patients before and after treatment

Since oxidative stress is involved in the development of many diseases[13], evaluating the thioredoxin (Trx) system as one of the main defence systems against oxidative stress can be very valuable. various studies have shown that the Trx molecule as a component of the redox system can play a role in many intracellular signalling pathways 
and the pathogenicity of some diseases [9, 14] To our knowledge, several studies have studied the serum level of Trx1 in schizophrenic patients, but this is the first report to investigate Trx1 gene expression in schizophrenic patients. In one of these studies, it was shown that serum Trx1 levels of first-episode schizophrenic patients were higher than that of the chronic patient and healthy controls; but there is no significant difference between chronic patients and healthy controls [10]. Our results were consistent with the results of this study. The results of our study show that the expression of the Trx1 gene in PBMCs of never medicated first schizophrenic patients was significantly higher than that of the healthy controls. The rate of Trx1 expression tended to be decreased after treatment with risperidone but was still higher than controls and this difference was not significant. The rate of Trx1 expression was a significant difference between patients after and before treatment. Based on the findings of this study, it appears that in schizophrenic patients, Trx1 gene expression is increased for unknown reasons, and the use of risperidone drug may modulate this increase. However, the Trx1 changes observed in the present study among the schizophrenic patients before and after treatment with risperidone was statistically significant, but confirmation of this requires further studies in larger populations.

If the study population is larger in future studies, statistically significant changes in Trx1 gene expression levels may be observed in patients with schizophrenia before and after treatment. In another study, Larsson et al. showed that a statistically significant difference among patients with the first episode of psychosis and long-term schizophrenia in comparison with control subjects, but there was no significant difference in plasma Trx1 between patients treated with typical and atypical neuroleptics, respectively within acute psychosis population [15]. Alper Bas et al. Showed that serum thioredoxin levels increased during acute psychosis and decreased during recovery [16]. In contrast to our study, Zhang et al showed that there was no significant difference in Serum TRX level between schizophrenic patients and healthy controls [17].

The results of our study also showed that, despite the hypothesis that oxidative stress may be a risk factor for cognitive impairment in schizophrenia and the possible role of thioredoxin as a compensatory or protective mechanism for neutralizing oxidative stress, no significant relationship was observed between the Trx1 gene expression in peripheral blood cells and positive and negative syndrome scale (PANSS) in schizophrenia. The results of other studies regarding the correlation between serum Trx1 and PANSS score were inconsistent [10, 15-17].

The brain is more susceptible to oxidative damage compared with other organs. Changes in Trx1 expression may lead to impaired redox homeostasis in the progression of neurodegenerative diseases and play a significant role in the pathogenicity of these diseases by disrupting the immune system [18]. It has been shown that the expression of mRNA and serum levels of Trex1 protein are increased in some autoimmune diseases such as rheumatoid arthritis, systemic lupus erythematosus, as well as in cancers and infections [9].

Results show that Trx-1 play an important role in inflammation and oxidative stress[14]. On the other hand, changes in serum levels of proinflammatory cytokines in patients with schizophrenia have been shown [19]. Therefore, changes in inflammatory cytokines in patients with schizophrenia may be associated with $\operatorname{Trx}-1$ expression and serum levels. Initially, loss of redox homeostasis and increased oxidative stress are involved in the pathogenesis of multiple sclerosis, and these two are stimuli for activating the brain stress response [13].

In Alzheimer's patients, Trx1 levels in brain tissue have been reported to be low [20] Also, numerous studies have shown changes in Trx expression in these patients [21]. In addition to determining the level of TRX1 expression in peripheral blood PBMCs of schizophrenic patients, another aim of this study was to determine the effect of risperidone on TRX1 expression in PBMCs of schizophrenic patients.

Preclinical studies have shown that typical antipsychotics are related to the oxidative system in the brain. But there is no clear relation between atypical antipsychotics and oxidative injury [22-24], On the other hand, Zhang et al. In a study showed that there is no association between the dose and duration of typical and atypical antipsychotic treatment with serum TRX level (17), also Larson et al. believe that antipsychotic treatment does not affect serum TRX levels [15].

In our study, the expression of TRX in PBMCs of schizophrenic patients decreased after risperidone treatment. This reduction in expression was statistically significant,and indicates the possible effect of risperidone on the expression of the TRX gene in PBMCs of these patients. Confirmation of the achievement of this study requires further research.

Acknowledgements: The authors wish to thank Psychiatrists in Taleghani Hospital in Ilam and Farabi Hospital in Kermanshah, for preparation of the blood samples. This study was financially supported by llam University of Medical Sciences (grant number: IR.MEDILAM.REC.1399.082).

Declaration of Conflicting Interests: All the authors declare that there is no conflict of interest for this project.

\section{REFERENCES}

1. Global, regional, and national incidence, prevalence, and years lived with disability for 354 diseases and injuries for 195 countries and territories, 1990-2017: a systematic analysis for the Global Burden of Disease Study 2017. Lancet (London, England). 2018;392(10159):1789-858.

2. Owen MJ. Implications of genetic findings for understanding schizophrenia. Schizophrenia bulletin. 2012;38(5):904-7.

3. van Os J, Kenis G, Rutten BP. The environment and schizophrenia. Nature. 2010;468(7321):203-12.

4. Reddy R, Keshavan M, Yao JK. Reduced plasma antioxidants in first-episode patients with schizophrenia. Schizophrenia research. 2003;62(3):205-12.

5. Mahadik SP, Evans DR. Is schizophrenia a metabolic brain disorder? Membrane phospholipid dysregulation and its therapeutic implications. The Psychiatric clinics of North America. 2003;26(1):85-102.

6. Finkel T, Holbrook NJ. Oxidants, oxidative stress and the biology of ageing. Nature. 2000;408(6809):239-47.

7. Watanabe R, Nakamura $H$, Masutani $H$, Yodoi J. Antioxidative, anti-cancer and anti-inflammatory actions by thioredoxin 1 and thioredoxin-binding protein-2. Pharmacology \& therapeutics. 2010;127(3):261-70. 
8. Lu J, Holmgren A. The thioredoxin antioxidant system. Free radical biology \& medicine. 2014;66:75-87.

9. Yoshihara E, Masaki S, Matsuo Y, Chen Z, Tian H, Yodoi J. Thioredoxin/Txnip: redoxisome, as a redox switch for the pathogenesis of diseases. Frontiers in immunology. 2014;4:514.

10. Zhang XY, Chen DC, Xiu MH, Wang F, Qi LY, Sun HQ, et al. The novel oxidative stress marker thioredoxin is increased in first-episode schizophrenic patients. Schizophrenia research. 2009;113(2-3):151-7.

11. Ogata FT, Batista WL, Sartori A, Gesteira TF, Masutani H, Arai RJ, et al. Nitrosative/oxidative stress conditions regulate thioredoxin-interacting protein (TXNIP) expression and thioredoxin-1 (TRX-1) nuclear localization. PloS one. 2013;8(12):e84588.

12. Javeri $A$, Ghaffarpour $M$, Taha MF, Houshmand $M$. Downregulation of miR-34a in breast tumors is not associated with either p53 mutations or promote hypermethylation while it correlates with metastasis. Medical oncology (Northwood, London, England). 2013;30(1):413.

13. Mahmood DFD, Abderrazak A, El Hadri K, Simmet T, Rouis M. The Thioredoxin System as a Therapeutic Target in Human Health and Disease. Antioxidants \& Redox Signaling. 2012;19(11):1266-303.

14. Shao R, Yang Y, Zhang Y, Zhao S, Zheng Z, Chen G. The expression of thioredoxin-1 and inflammatory cytokines in patients with sepsis. Immunopharmacology and Immunotoxicology. 2020;42(3):280-5.

15. Owe-Larsson B, Ekdahl K, Edbom T, Osby U, Karlsson H, Lundberg $\mathrm{C}$, et al. Increased plasma levels of thioredoxin-1 in patients with first-episode psychosis and long-term schizophrenia. Prog Neuropsychopharmacol Biol Psychiatry. 2011;35(4):1117-21.

16. Baş A, Gultekin G, Incir S, Bas T, Emul M, Duran A. Level of serum thioredoxin and correlation with neurocognitive functions in patients with schizophrenia using clozapine and other atypical antipsychotics. Psychiatry Research. 2016;247.

17. Zhang XY, Chen DC, Xiu MH, Yang FD, Tan YL, He S, et al. Thioredoxin, a novel oxidative stress marker and cognitive performance in chronic and medicated schizophrenia versus healthy controls. Schizophrenia research. 2013;143(2):3016.

18. Pennisi G, Cornelius C, Cavallaro MM, Salinaro AT, Cambria MT, Pennisi M, et al. Redox regulation of cellular stress response in multiple sclerosis. Biochemical Pharmacology. 2011;82(10):1490-9.

19. Azizi E, Hosseini A, Soudi S, Noorbala A, Bastaminejad S. Evaluation of Immunologic Function of Peripheral Blood Monocytes from Schizophrenic Patients in Response to Toxoplasma Gondii. Clinical and Experimental Investigations. 2020:1-8.

20. Maulik N, Das DK. Emerging potential of thioredoxin and thioredoxin interacting proteins in various disease conditions. Biochimica et Biophysica Acta (BBA) - General Subjects. 2008;1780(11):1368-82.

21. Jia J, Zeng X, Xu G, Wang Z. The Potential Roles of Redox Enzymes in Alzheimer's Disease: Focus on Thioredoxin. ASN Neuro. 2021;13:1759091421994351.

22. Abdel-Sattar EA, Mouneir SM, Asaad GF, Abdallah HM Protective effect of Calligonum comosum on haloperidolinduced oxidative stress in rat. Toxicology and Industrial Health. 2012;30(2):147-53.

23. Parikh V, Khan MM, Mahadik SP. Differential effects of antipsychotics on the expression of antioxidant enzymes and membrane lipid peroxidation in rat brain. Journal of Psychiatric Research. 2003;37(1):43-51.

24. Pillai A, Parikh V, Terry AV, Mahadik SP. Long-term antipsychotic treatments and crossover studies in rats: Differential effects of typical and atypical agents on the expression of antioxidant enzymes and membrane lipid peroxidation in rat brain. Journal of Psychiatric Research. 2007;41(5):372-86 